\title{
La formación del profesorado universitario de ciencias. El conocimiento didáctico y la investigación científica
}

\author{
María Gabriela Lorenzo ${ }^{1,2, a}$, Andrea Soledad Farré ${ }^{1 b}$, Alejandra María Rossi ${ }^{1 \mathrm{c}}$ \\ ${ }^{1}$ Universidad de Buenos Aires. Facultad de Farmacia y Bioquimica. Centro de Investigación y Apoyo a la \\ Educación Cientifica (CLAEC). Ciudad autónoma de Buenos Aires. Argentina. \\ ${ }^{2}$ Consejo Nacional de Investigaciones Científicas y Técnicas (CONICET) \\ "glorenzo@ffyb.uba.ar, basfarre@ffvb.uba.ar,도ssialejandramaria@gmail.com
}

[Recibido: 18 Abril 2017. Revisado: 24 Enero 2018. Aceptado: 7 Abril 2018]

\begin{abstract}
Resumen: En la actualidad ha aumentado el interés por desarrollar nuevas estrategias didácticas para la enseñanza de las ciencias experimentales. Así, para enseñar una visión contextualizada y provisional de la ciencia, los conocimientos sobre su naturaleza deben hallarse estrechamente relacionados con los conocimientos didácticos de los contenidos a enseñar. En este trabajo se plantea un dispositivo didáctico sobre la naturaleza del trabajo de investigación científica con el fin de contribuir con la formación del profesorado universitario del campo de las ciencias de la salud desde la perspectiva de la investigación-acción. Se presentan los criterios que guiaron su diseño y los resultados del análisis de su implementación. Los resultados muestran que los docentes universitarios sostenían imágenes de una ciencia de características hipotético-deductivas aunque provisorias, y que basaban su enseñanza en ideas mayormente intuitivas. El trabajo en pequeños grupos y el debate en plenario permitieron interpelar sus representaciones, reflexionar sobre las imágenes de ciencia, propias y ajenas, así como revisar posibles estrategias de enseñanza fundamentadas teóricamente.
\end{abstract}

Palabras clave: Conocimiento Didáctico del Contenido; Naturaleza de la Ciencia; Enseñanza Universitaria; Formación de Profesores.

University Science teachers' education. Pedagogical knowledge and scientific research.

\begin{abstract}
The interest in the development of new pedagogical strategies for experimental science teaching have increased these days. Thus, in order to teach a contextualized and provisional image of science, the nature of science knowledge must be linked with the pedagogical content knowledge to be taught. A pedagogical proposal about the nature of the scientific research is posed in this work to contribute with university teachers' education in the field of Health Sciences using action research model. Criteria for the design and the analysis of enacting results are presented here. The results show that university teachers uphold hypothetic-deductive images about Science but these were provisional, and they based their teaching on mainly intuitive ideas. The work in small groups and plenary debate allowed to confront teachers' representations, to reflect about own and others' images of science, as well as to review possible theoretical founded teaching strategies.
\end{abstract}

Keywords: Pedagogical Content Knowledge, Nature of Science, High Education, Teachers Training

Para citar este artículo: Lorenzo, M.G., Farré, A.S., Rossi, A.M (2018) La formación del profesorado universitario de ciencias. El conocimiento didáctico y la investigación científica. Revista Eureka sobre Enseñanza y Divulgación de las Ciencias 15(3), 3603. doi: 10.25267/Rev_Eureka_ensen_divulg_cienc.2018.v15.i3.3603

\section{Introducción: Los profesores y la enseñanza universitaria}

Los aspectos investigados por la didáctica de la ciencia fueron cambiando a lo largo del tiempo. En 1994, Carles Furió nos advertía sobre el corrimiento que en el futuro tomarían estas investigaciones orientadas hacia el desarrollo profesional docente: «... se está pasando de investigar lo que piensa y hace el alumno en clase hacia lo que piensa y hace el profesor, tratando de analizar su actividad y así poder descifrar las claves de su desarrollo profesional. En particular, habrá que determinar cuáles son sus necesidades formativas y qué factores pueden influir en una continua preparación para el cambio didáctico» (Furió 1994, p. 188). Hoy sus palabras han demostrado ser acertadas y, desde entonces, los estudios sobre el conocimiento del profesor han tomado gran relevancia fundamentalmente para los profesores 
de primaria y secundaria, aunque no del mismo modo para la formación del profesorado universitario.

La evaluación de la calidad de las universidades por organismos nacionales e internacionales, las reformas universitarias y las evaluaciones de los docentes de las últimas décadas, han abierto nuevas discusiones al resaltar la necesidad de formación pedagógica del profesorado universitario (Briceño, Benarroch y Marín 2013, Mas 2011), siendo su desarrollo profesional un elemento clave para la calidad de la enseñanza universitaria. En este sentido, De Miguel (2003) alude a la responsabilidad que tienen las universidades públicas ante la sociedad de asegurar los procedimientos de selección y formación del profesorado para garantizar una enseñanza de calidad. No obstante, los trabajos que informan sobre esta problemática son muy escasos; por ejemplo, desde el 2012 no ha habido reportes sobre la formación de profesores universitarios en esta revista.

Los estudios en el marco del Espacio Europeo de Educación Superior sobre las necesidades de formación en competencias docentes (Álvarez-Rojo et al. 2011) y aquellos que propician la inclusión de aspectos históricos de la disciplina (García-Martínez e Izquierdo, 2014) pueden servirnos de referencia para el contexto latinoamericano. Igualmente lo hacen aquellos en los que se reconocen las dificultades de los docentes (universitarios) para transferir, transponer o aplicar los contenidos supuestamente aprendidos en los cursos convencionales; y, los que proponen sistemas de mentorías para que un acompañamiento más prolongado que los ayude a reflexionar sobre sus prácticas y sus creencias sobre la enseñanza y el aprendizaje (Barragués et al., 2014).

El desarrollo profesional de los docentes es una actividad compleja que no puede pensarse alejada de la integración entre instancias metacognitivas que incluyan los aspectos personales, disciplinares, contextuales y la propia práctica (Vázquez, Jiménez y Mellado 2007) porque, como advertía Furió (1994), no tiene sentido esperar cambios en las prácticas de los profesores en servicio por su mera participación en cursos de capacitación docente, cuya propuesta sea «una yuxtaposición de saberes académicos que hay que aplicar en la práctica» (p. 189).

A diferencia de otros niveles, la formación del profesorado universitario no suele estar sistematizada. Desde los orígenes de la universidad pareció aceptarse que el conocimiento disciplinar sobre la asignatura era condición necesaria y suficiente para poder enseñarla. Es decir que para el ejercicio de la docencia en la universidad lo fundamental sería la formación en el campo específico. Hoy existen razones para cuestionarnos sobre esta forma de entender a los estudios superiores y, por ende, la formación de sus docentes (Lorenzo 2017). Para dar respuestas a estas nuevas demandas y necesidades, muchas universidades han comenzado a tomar conciencia de la situación, reflexionando e iniciando algunos procesos de transformación. Así, se han planteado diferentes cuestionamientos (Imbernón 2011) y dispositivos de capacitación, que, con diverso éxito, propenden al desarrollo de las competencias de los docentes universitarios (Zabalza 2007).

Dado el interés por desarrollar nuevas estrategias didácticas en ciencias experimentales para la formación inicial del profesorado (Jiménez-Tenorio y Oliva 2016), este trabajo aborda el estudio de la formación en activo de profesores universitarios de ciencia.

\section{Marco Teórico}

Además de los procesos de enseñanza generales en el nivel superior (Grisales-Franco 2012), al reflexionar sobre la formación didáctica del profesorado universitario de ciencias experimentales, Mellado (1999) destaca la necesidad de una didáctica específica que considere 
principalmente la disciplina o la ciencia referente (Moreno 2011). Si bien, las investigaciones reportadas para otros niveles o disciplinas pueden servir como base, no pueden extrapolarse directamente a la enseñanza universitaria de ciencias experimentales.

En la bibliografía, acerca de las concepciones sobre la naturaleza de la ciencia de profesores universitarios, una investigación realizada sobre un grupo de trece profesores chilenos establece una correlación entre la visión empirista de ciencia y la visión de transmisiónrecepción del aprendizaje (Martínez y González 2014). En tanto, sobre el conocimiento profesional de profesores universitarios, Jaruta y Medina (2012) presentan un estudio de casos de tres profesores considerados excelentes para mostrar la complejidad del conocimiento profesional para la enseñanza universitaria. El rasgo saliente de dichos profesores es haber ejercido la docencia por más de dieciocho años. Merece entonces plantearse la posibilidad de instrumentar dispositivos que permitan acortar ese tiempo y trascender el aprendizaje basado en la propia experiencia, a partir de una propuesta sistemática de formación del profesorado.

En base a estos antecedentes proponemos una actividad pensada para optimizar ese tiempo por medio de la reflexión sobre la práctica, la toma de conciencia y revisión de las propias ideas. Tomamos como modelo teórico y metodológico el programa del Conocimiento Didáctico del Contenido -CDC- (Shulman, 1986) de mostrada utilidad para el estudio del conocimiento profesional docente (Lorenzo, Daza y Garritz 2014). El contenido temático seleccionado fue la naturaleza del trabajo de investigación científica, hasta ahora inexplorado desde la didáctica de las ciencias, considerando los aportes de las corrientes que abordan la Naturaleza de la Ciencia -NdC-(Acevedo 2009a, 2009b, Lederman 2006), dado que «...es imprescindible hacer un tratamiento explícito y reflexivo de los contenidos de $\mathrm{NdC}$ en los programas de formación del profesorado de ciencias» (Acevedo 2010, p. 656).

Los debates desde la tradición Ciencia-Tecnología-Sociedad sobre la incorporación de la NdC a la enseñanza, la selección de contenidos y las distintas formas de enseñarlo (Acevedo-Díaz y García-Carmona 2016) así como sobre las posibles articulaciones con el CDC, para los niveles primario y secundario de la educación (Demirdöğen et al., 2016), resultan necesarios pero no suficientes para abordar la problemática de la enseñanza de las ciencias en el nivel universitario.

El CDC es un conocimiento complejo que se construye a lo largo de la vida del profesor, donde se combinan la formación disciplinar con otros conocimientos (que devienen de la historia, la epistemología y las implicaciones sociales y tecnológicas de la propia disciplina); pero además, incluye los conocimientos relacionados con la enseñanza y el aprendizaje de los contenidos en el contexto donde se desempeña. Es así que el CDC es el conocimiento que posibilita la transformación de la lógica disciplinar en la lógica de la enseñanza y la adaptación que los profesores construyen de un contenido para enseñarlo. La definición inicial del CDC, que lo describe como una amalgama entre el conocimiento disciplinario y el conocimiento pedagógico del profesor, sigue siendo esclarecedora; ya que este conocimiento le permite al profesor desarrollar con éxito su tarea de enseñar eligiendo los mejores ejemplos, analogías, demostraciones y otras estrategias didácticas para un determinado grupo de alumnos.

El programa original sobre el CDC como modelo de cognición docente y su documentación ha comenzado a emplearse en la formación de docente tanto para investigar el conocimiento de los futuros docentes y, también, como estrategia formativa (Berry, Depaepe y van Driel 2016). Específicamente en la formación de docentes de Ciencias Naturales, el modelo más empleado ha sido el de Magnusson, Krajcik y Borko (1999) que considera al CDC conformado por cinco dominios de conocimientos y creencias sobre: 1) los modelos de enseñanza de las ciencias, 2) el contenido (aspectos semánticos y sintácticos) y las razones por las cuales los estudiantes deben aprenderlo, 3) el aprendizaje de los estudiantes (concepciones 
alternativas y dificultades), 4) las estrategias didácticas que puedan emplearse para enseñar el contenido, y 5) los aspectos importantes a ser evaluados y las estrategias utilizadas.

Basándose en este modelo, el instrumento privilegiado para la documentación del CDC en Latinoamérica (Lorenzo et al. 2014) ha sido el cuestionario de la Representación del Contenido (ReCo) ideado por Loughran, Mulhall y Berry (2004). Este mismo instrumento también ha sido utilizado para el desarrollo del CDC ya que permite a los docentes reconocer los dominios del CDC, relacionarlos con la práctica áulica problematizando el contenido y las estrategias didácticas empleadas, de una forma diferente a la que se realiza generalmente en las capacitaciones tradicionales (Berry et al. 2016).

Por su parte, la $\mathrm{NdC}$ se destaca como un eje esencial para la formación del profesorado de todos los niveles de enseñanza porque permite mostrar a la ciencia como una actividad humana, sujeta a cambios y controversias (Lederman, Antink y Bartos 2012). En el ámbito universitario, muchos de los profesores realizan tareas de investigación científica. Sin embargo, no es habitual que hayan tenido la ocasión de reflexionar sobre la propia naturaleza de la disciplina en la que se desempeñan (Faikhamta 2013). Esta carencia de pensamiento metacientífico y metacognitivo podría interpretase, en un sentido khuniano, debida a la invisibilidad del paradigma en el cual desarrollan sus actividades científicas, que sumado a su misma inconmensurabilidad, dificulta (cuando no impide) ver más allá de lo conocido o mirar lo conocido de otras maneras posibles. Estas son razones poderosas que justifican la inclusión del tópico naturaleza de la ciencia como parte trascendental en la formación pedagógica de los profesores universitarios. Simultáneamente, pone en evidencia la relevancia de la didáctica de las ciencias al integrar en un mismo corpus de conocimiento la ciencia, su naturaleza y los aspectos asociados a su enseñanza y aprendizaje.

Dado que la literatura ha mostrado que entre los docentes universitarios de ciencia predomina cierta indiferencia hacia cualquier conocimiento de carácter pedagógico y didáctico (Briceño y Benarroch 2013) para poder diseñar actividades que resulten significativas deben tomarse en cuenta las concepciones y creencias sobre el propio rol del profesorado (Martín del Pozo, Porlán Rivero. 2005). No obstante, el mero conocimiento de dichas ideas no resuelve el problema de la transferencia al aula para una enseñanza desde una visión de ciencia contextualizada (Lorenzo y Farré 2014). Esto puede entenderse como una derivación de la propia estructura que poseen las ofertas de formación de los profesores. Si los contenidos metadisciplinares aparecen separados e inconexos de los disciplinares, a los docentes se les dificultará pensar en estrategias para abordar la naturaleza de la ciencia al enseñar los contenidos disciplinares específicos de su materia (Acevedo et al. 2005).

Nuestra labor docente concierne a la formación y capacitación de docentes universitarios en el campo de la didáctica de las disciplinas específicas de las ciencias naturales y de la salud (Lorenzo 2012). La enseñanza universitaria en este ámbito ha sido concebida durante mucho tiempo, como un ejercicio mecánico y repetitivo de trasmisión de conocimientos, sin tener en cuenta la capacidad creadora del ser humano ni los conocimientos que, sin lugar a duda, poseen los estudiantes cuando inician estudios universitarios. Esto ha implicado siempre un desafío a la hora de proponer dispositivos de intervención basados en un enacting o puesta en acción sobre la manera de llevar a la práctica áulica esos conocimientos disciplinares específicos que resulten a la vez atractivos y formativos para un colectivo con alto nivel de conocimientos disciplinares y formación heterogénea en cuestiones pedagógicas. Para dar respuesta a esta situación se implementó una secuencia didáctica basada en el CDC como modelo formativo llevada a cabo en un programa de formación inicial en docencia universitaria. Los objetivos de este trabajo son presentar los criterios que guiaron su diseño y los resultados del análisis de su implementación desde la perspectiva de investigación-acción 
acerca del conocimiento didáctico sobre el trabajo de investigación científica de docentes universitarios.

\section{Metodología}

\section{Contexto y participantes}

La secuencia fue incluida en la asignatura Didáctica y Epistemología de las Ciencias de la Salud perteneciente a la Carrera Docente de la Facultad de Farmacia y Bioquímica de la Universidad de Buenos Aires. Esta carrera, de dos años de duración, está destinada a la formación didáctica del personal docente en actividad de la propia institución, y se ofrece de manera optativa sin costo alguno para los docentes.

Los alumnos de la asignatura son docentes con formación específica en la disciplina que enseñan y amplios conocimientos específicos, dominio de la bibliografía y de los procedimientos de la investigación científica en su campo, avalados por diversos mecanismos institucionales. Participaron 59 docentes graduados universitarios de carreras asociadas a las ciencias de la salud (farmacéuticos, bioquímicos, médicos, biólogos) que impartían clase, en un total de 27 asignaturas pertenecientes a grandes áreas del conocimiento: Física (Física, Óptica, Metodología de radioisótopos), Química (Orgánica, Analítica, Inorgánica, Biológica, Físicoquímica), otras asociadas con la Biología (Fisiopatología, Microbiología, Nutrición, Inmunología, Virología) y otras específicas de las carreras (Farmacobotánica, Biotecnología, Bioquímica Clínica, Cinética Enzimática, Genética Diagnóstica y Forense, Farmacología, Toxicología y Química Legal, Farmacia Clínica y Asistencial, Tecnología Farmacéutica).

\section{Diseño e implementación de la secuencia}

Con el fin de favorecer una acción formativa que permitiera a su vez indagar en las concepciones de los profesores universitarios sobre el trabajo de investigación científica desde la perspectiva de la naturaleza de la ciencia, se diseñó una secuencia didáctica que incluyó distintas tareas y actividades combinadas. La implementación de la secuencia alternó trabajo individual, en equipos y plenarios. Por equipo entendemos un grupo cuyos integrantes trabajan juntos y en forma cooperativa en pos de conseguir una meta u objetivo común (Huertas y Montero 2001, Lorenzo 2009). De este modo, se promueve la interacción entre pares para la construcción conjunta de conocimientos.

La secuencia se basó en una adaptación del cuestionario ReCo (Figura 1). En un primer momento, se presentó como una actividad de introducción al tema con el fin de generar los insumos necesarios para el desarrollo del segundo encuentro. 


\section{PARA SABER MÁS SOBRE NUESTRA PROPIA PRÁCTICA}

Las asignaturas que se dictan en las carreras de Farmacia y de Bioquímica, en su mayoría, se encuentran fuertemente vinculadas con el trabajo científico, tanto es así que muchos de los docentes se desempeñan como investigadores. A partir de las preguntas que se presentan a continuación, intentaremos reflexionar en grupos de cátedra o por afinidad de asignaturas sobre el impacto de nuestro trabajo como investigadores científicos en nuestras prácticas de enseñanzas y la naturaleza de esa relación.

Sobre el trabajo científico y nuestra enseñanza...

1. ¿Qué es lo que intentan (explícita o implícitamente) que sus estudiantes aprendan sobre la naturaleza del trabajo científico, en su disciplina en particular?

2. ¿Por qué creen que sería importante para los estudiantes aprender sobre esto?

3. ¿Qué otras cosas saben Uds. sobre la investigación científica que consideran que todavía no es el momento para enseñárselas a sus estudiantes?

4. Cuáles son las dificultades/limitaciones relacionadas con la enseñanza de esta idea?

5. ¿Qué saben Uds. sobre la forma de pensar de sus alumnos que influyen su forma de enseñar esta idea?

6. ¿Qué otros factores influyen en la enseñanza de esta idea?

7. ¿Utilizan alguna estrategia para enseñar sobre el trabajo científico? ¿Cuáles? ¿Por qué eligen trabajarlo de esa manera?

8. Si tuvieran que evaluar la comprensión que los estudiantes tienen sobre el trabajo científico (cada uno en su asignatura) ¿Cómo lo harían?

Figura 1. ReCo utilizado para la indagación del CDC, adaptado de Loughran et al. (2004)

Primer momento. Se destinó la última hora de una clase para responder el cuestionario en pequeños grupos por afinidad temática a partir de la discusión entre pares. Las respuestas obtenidas fueron recolectados y analizadas por el equipo docente.

Segundo momento. A la siguiente clase las respuestas dadas al cuestionario de la ReCo se retomaron recursivamente.

I. Indagación y explicitación de concepciones e ideas previas:

a) Análisis de las propias respuestas. En los mismos grupos de trabajo, una semana después, cada equipo recibió el cuestionario con sus respuestas para releerlo y revisarlo. Así, pudieron revisar sus respuestas.

b) Análisis de las ReCo de otros grupos. Cada equipo reflexionó sobre las respuestas de sus compañeros, compararlas y discutirlas al interior de cada grupo.

c) Puesta en común en plenario. La totalidad de las respuestas y reflexiones aportadas por los distintos grupos se presentó oralmente.

d) Contrastación de las propias respuestas con las reflexiones aportadas durante el plenario. Al interior de cada uno de los grupos originales se propició la reflexión sobre sus respuestas a partir de contar con una mayor cantidad de puntos de vista. Así, pudieron puntualizarse las ideas compartidas y las particularidades entre todo el grupo en su conjunto.

e) Finalmente, se incorporó al debate colectivo el aporte de los resultados encontrados por el análisis realizado por el equipo docente sobre las respuestas de la primera clase, a modo de preguntas o cuestiones que obligaran a nuevas reflexiones sobre las respuestas dadas. Esto permitió elaborar una conclusión general en plenario. 
II. Introducción de nueva información sobre el tópico "Corrientes epistemológicas del siglo XX” (Popper, Lakatos, Khun, Bachelard, Feyerabend). Durante la exposición se retomaron las respuestas vertidas en la ReCo para promover una redescripción de las representaciones de los participantes. Las cuestiones explicitadas durante esta actividad fueron posteriormente discutidas desde los marcos teóricos aportados por los diferentes modelos epistemológicos a partir de la lectura de bibliografía específica. De este modo, se favoreció una mayor comprensión por parte de los cursantes de sus propias posturas, al reconocerse en las diferentes corrientes epistemológicas y, por lo tanto, generando nuevas oportunidades para reflexionar sobre sus concepciones.

III. Actividades metacognitivas: Los últimos 40 minutos de la clase se destinaron a revisar y reflexionar sobre lo realizado. Esta actividad forma parte habitual en el desarrollo de este curso de formación docente y con ella se pretende transitar por dos dimensiones diferentes, aunque complementarias:

a) La propia experiencia como alumno del curso respondiendo a las siguientes preguntas:

- ¿Qué aprendí a lo largo de esta clase?

- ¿Cómo lo aprendí?

- ¿Qué dificultades se me presentaron?

- ¿En qué creo que cambiará mi forma de organizar y dar mis clases después de haber realizado esta actividad?

Luego, se les ofreció la oportunidad, de compartir alguna de sus reflexiones con sus compañeros.

b) La reflexión que como docentes de su propia asignatura pudieran realizar a partir de la experiencia vivenciada en la clase (Figura 2).

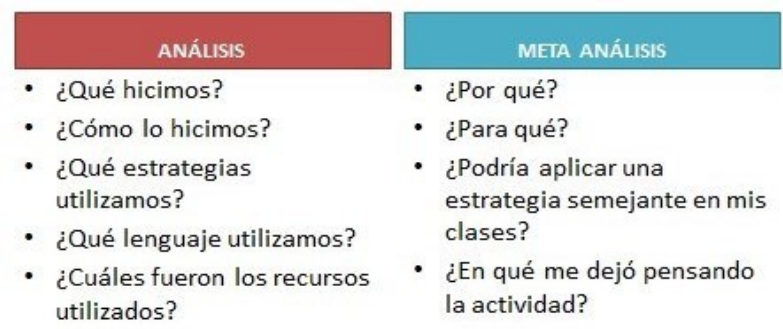

Figura 2. Actividad metacognitiva: Hagamos un cierre...

\section{Diseño y análisis de datos}

Los datos fueron recogidos durante la implementación de la secuencia desde una perspectiva de investigación-acción (Latorre 2005) en el marco de una investigación educativa críticamente comprometida con la transformación de las prácticas convencionales a partir de la generación de nuevo conocimiento. Se analizaron cualitativamente, las respuestas al cuestionario ReCo correspondientes a 16 grupos de entre 2 a 6 participantes, empleando la teoría fundamentada (Glaser y Strauss 1967) para el establecimiento de categorías. Los resultados fueron triangulados con el registro de las observaciones no participantes. 


\section{Resultados y Discusión}

Las respuestas al cuestionario ReCo pudieron agruparse en tres dimensiones: a) sobre la naturaleza del trabajo científico, b) sobre la enseñanza del y sobre el trabajo científico, y c) sobre las estrategias de enseñanza y de evaluación. En el texto incluimos entrecomilladas algunas frases textuales de los participantes a modo de ejemplo. Luego se detalla como estas visiones iniciales cambiaron a partir de la intervención didáctica.

\section{De la naturaleza del trabajo científico}

Las tres primeras preguntas hacen referencia al conocimiento sobre la temática teniendo en cuenta la intencionalidad de la enseñanza, la importancia atribuida y aquellos otros conocimientos que saben los docentes pero que por diferentes razones no son tenidos en cuenta en sus clases. Aunque existió una gran dispersión en las respuestas, pudimos detectar algunas ideas predominantes.

Los docentes dijeron intentar que sus estudiantes aprendieran primordialmente la naturaleza experimental del trabajo científico (13\% de las respuestas), entendiéndose como experimental a la aplicación de "el método científico" y todas sus etapas, desde el planteo y validación de hipótesis hasta la elaboración de informes. Merece destacarse la importancia dada a "el análisis crítico de los resultados" (12\% de las respuestas). Con menor frecuencia aparecieron la búsqueda y revisión de la bibliografía, y, la naturaleza dinámica y el carácter provisional del conocimiento científico (Figura 3).

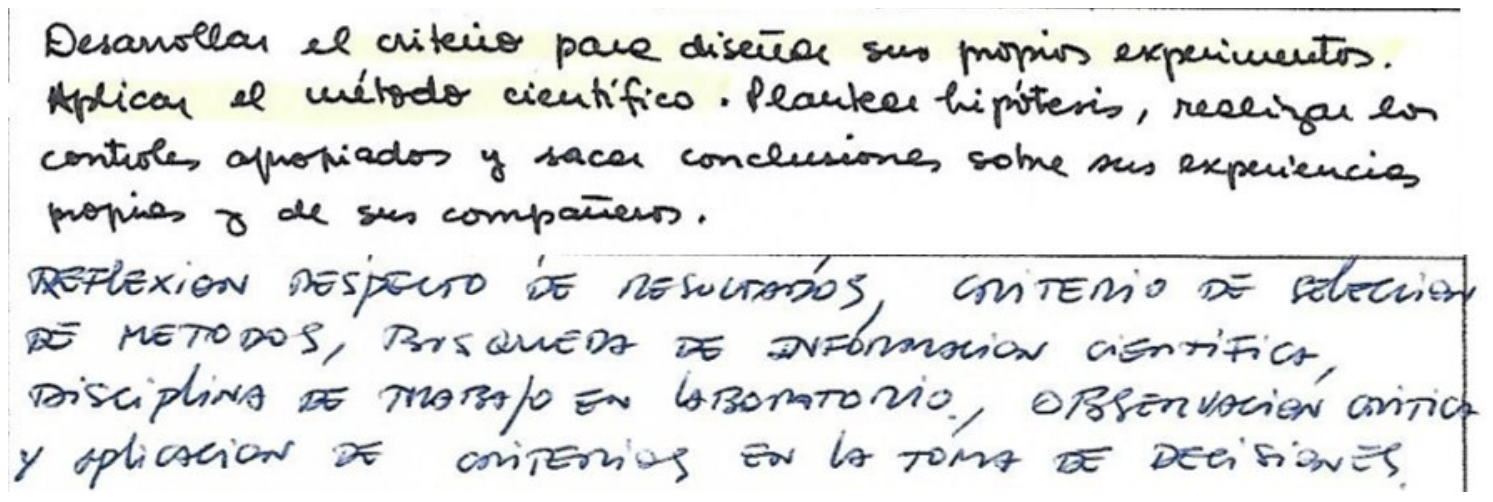

Figura 3

La importancia dada a la enseñanza de cuestiones concernientes con el trabajo científico, estuvo influenciada por el contexto. El 20\% de las respuestas estuvieron relacionadas con las incumbencias profesionales (actividades reservadas para la titulación académica) y las posibles actividades a desarrollar en el campo laboral por los egresados de la institución (Figura 4).

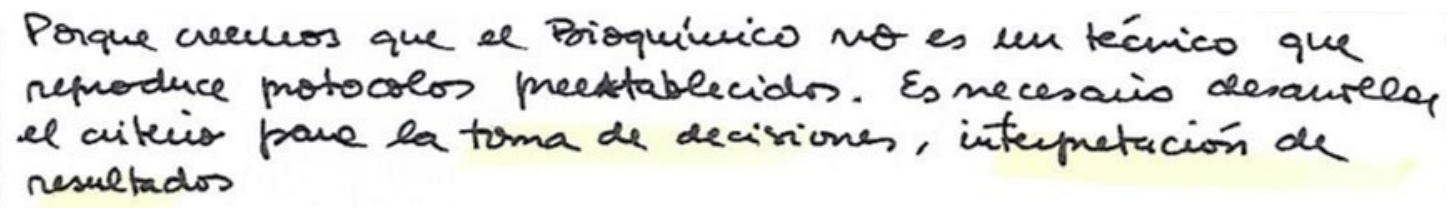

Figura 4

Otras respuestas hicieron referencia a una variedad de temas, como la interrelación entre ciencia, tecnología y sociedad, las implicaciones éticas y morales del trabajo científico, el carácter colaborativo de la ciencia, o el compartir con los estudiantes sus propias experiencias. Es interesante destacar la respuesta de uno de los grupos (\#9, conformado por docentes de química) en la que resaltaron la importancia de que los alumnos disfrutaran mientras realizan 
el trabajo experimental. Otros grupos destacaron la importancia del tema como motivador o generadores de interés para los estudiantes.

Al responder sobre los conocimientos que quedaban por fuera de su enseñanza hubo respuestas que variaron desde "nada" a "todo". No obstante, un gran número de ellas se refirieron a las peculiaridades del sistema científico que incluían cuestiones tales como los mecanismos no siempre claros para lograr una publicación, las dificultades para obtener financiamiento para los proyectos de investigación, la burocracia de los organismos de ciencia y tecnología, los salarios y las evaluaciones constantes a las que son sometidos los investigadores y sus producciones. Otros mencionaron explícitamente las dificultades y el esfuerzo que requiere la investigación científica (Figura 5).

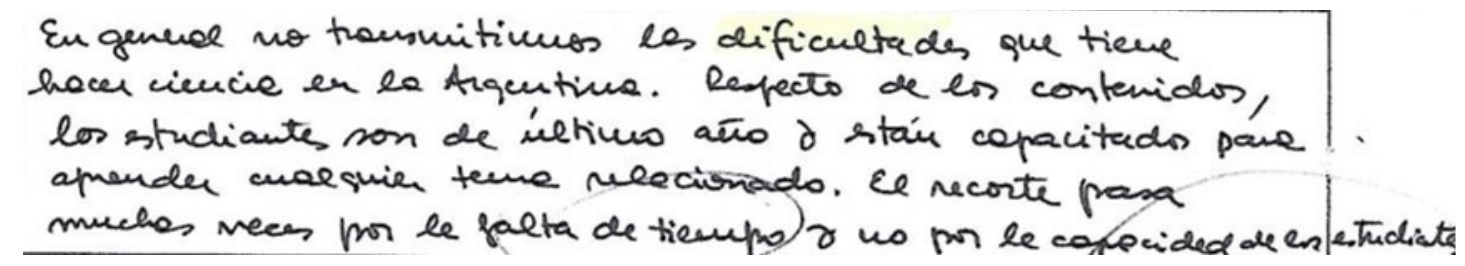

Figura 5

\section{Dificultades en la enseñanza del trabajo científico}

Las preguntas 4, 5 y 6 del cuestionario fueron respondidas de manera semejante por los participantes. El análisis de las respuestas mostró que las dificultades son atribuidas exclusivamente a factores externos, donde el docente se parece más a un espectador que a un actor de la escena educativa. Los obstáculos mencionados son de tipo general aplicables a más de un tópico (Figura 6).

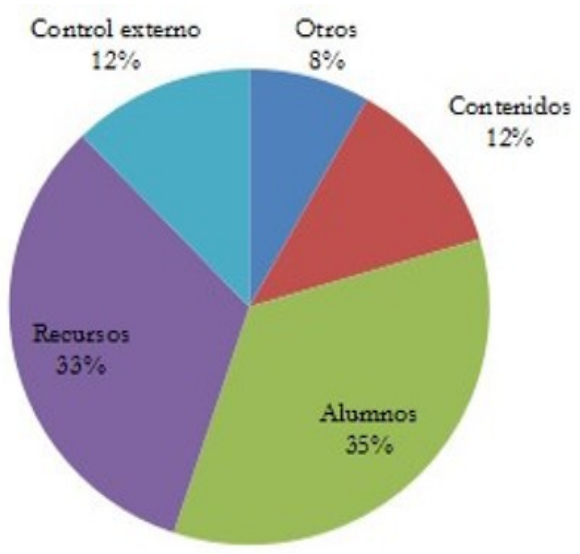

Figura 6. Principales dificultades y factores que influyen en la enseñanza del tópico

Mayoritariamente las dificultades se atribuyen a los alumnos como falta de... de interés, de motivación, de inquietud, de capacidad de asombro, de pensamiento crítico, de competencia práctica, de preparación o de conocimientos previos, de visiones que poseen que no se corresponden con lo que es la ciencia y los científicos y la dificultad que implica su cambio. Otras razones refieren a aspectos organizacionales como la cantidad de estudiantes por grupo de clase (Figura 7). En pocas ocasiones se mencionaron aspectos positivos sobre conocimientos que ya poseen los estudiantes como ser su "permeabilidad" para las nuevas tecnologías. Mientras, otras limitaciones fueron atribuidas la falta de cuestiones materiales, 
bajos recursos económicos, equipamiento insuficiente ("limitantes tecnológicas") y falta de tiempo.

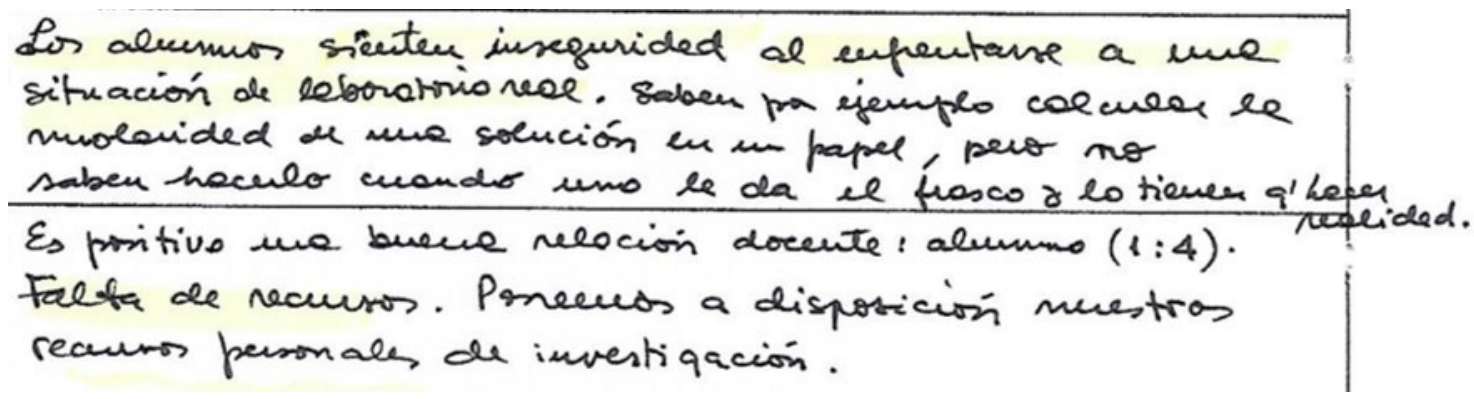

Figura 7

También mencionaron dificultades debidas a los contenidos, ya sea por su cantidad, complejidad o por la ubicación de la asignatura en el plan de estudios; presumiblemente conectado con el control externo, planteado como "riesgo de alejarse de lo pautado", a lo establecido en el programa de la asignatura. En las discusiones posteriores se pudo correlacionar estas respuestas con la convicción de los docentes de ceñirse al currículum prescripto y al temor derivado de su incumplimiento frente a las autoridades académicas.

De modo incipiente aparecieron otros aspectos que muestran un posicionamiento más crítico frente a la enseñanza del tópico como "la falta de diálogo entre las asignaturas" y "la experiencia del docente". Además, mencionaron la postergación del inicio de las actividades de investigación para los estudiantes de grado y la falta de opciones para elegir entre las investigaciones disponibles en la institución. Durante la discusión colectiva se pusieron en cuestión las posibilidades concretas de los estudiantes para incorporarse a grupos de investigación a través del programa de becas o proyectos de investigación financiados o como auxiliares de docencia y de investigación.

\section{De las estrategias de enseñanza y de evaluación}

La pregunta 7 refiere a las estrategias con las que los docentes enseñan sobre el trabajo científico, mientras que la pregunta 8 alude a las formas de evaluación.

Como estrategias principales para la enseñanza del trabajo científico (Figura 8) se encontraron los aportes del docente para la discusión basados en ejemplos de casos reales o ficticios, discusión de resultados de trabajos propios o ajenos, simulaciones y el relato de la propia experiencia como investigadores. La búsqueda y discusión de artículos (papers) de la especialidad fue otra estrategia importante. En menor medida, aparecieron la estrategia llamada póster, planteada como una especie de juego de roles donde los estudiantes simulan participar de un congreso y exponen los resultados de su trabajo a través de una lámina, seguida por la experimentación y la realización de cálculos numéricos. 


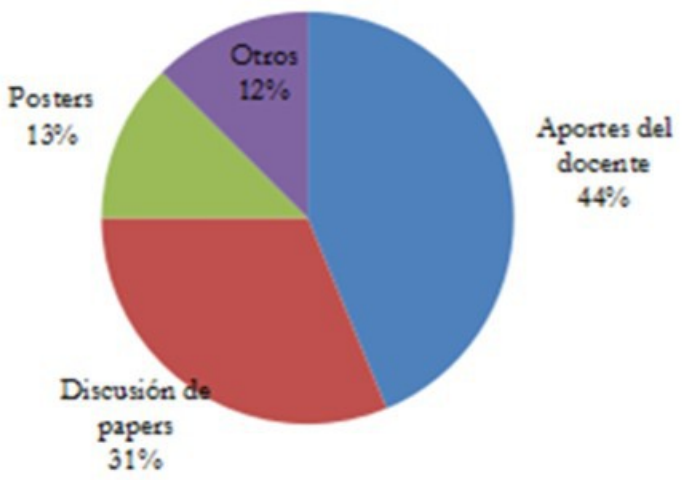

Figura 8. Estrategias para la enseñanza sobre el trabajo científico

Si bien la pregunta 7 era más amplia, inicialmente los docentes no respondieron a la cuestión “¿por qué eligen trabajarlo de esa manera?" Por lo que este aspecto fue retomado en la discusión grupal. A partir de la reflexión posterior realizada, los propios docentes sobre sus respuestas, surgió la influencia de la tradición y las costumbres heredadas, evidenciando la importancia que tiene el contexto académico en la generación y legitimación de la identidad profesional del docente (Caballero y Bolívar 2015). Asimismo, resaltaron la relevancia de las innovaciones, la "simulación de congresos" y los pósteres, como resultado de los cursos de formación pedagógica realizados previamente por otros miembros del equipo docente de la asignatura, reconociendo la relevancia de pertenecer a una determinada comunidad de aprendizaje (Liesa, Castelló y Becerril 2018).

Las principales estrategias ofrecidas para la evaluación de los estudiantes mostraron una concepción cientificista correlacionando el resultado del aprendizaje con los resultados de investigación (Figura 9). Así, propusieron la realización de un proyecto de trabajo grupal, cuya extensión podía variar desde algunas pocas clases a toda la asignatura, considerada como una "actividad integradora" y que finalice con una exposición oral o puesta en común a través de la realización de un artículo o póster. Otras estrategias semejantes quedaron circunscritas a ciertos aspectos del trabajo científico, como la búsqueda y análisis de bibliografía, el diseño de protocolos para el trabajo experimental o el análisis de resultados experimentales. También propusieron el análisis de casos o situaciones problemáticas y se mencionaron los exámenes orales y la evaluación a través de la observación del desempeño de los estudiantes durante las prácticas de laboratorio. A partir de la discusión en los grupos se evidenció el carácter intencional de muchas de las respuestas a esta pregunta, ya que el trabajo científico no era un tópico que fuera enseñando, y mucho menos evaluado, explícitamente. 


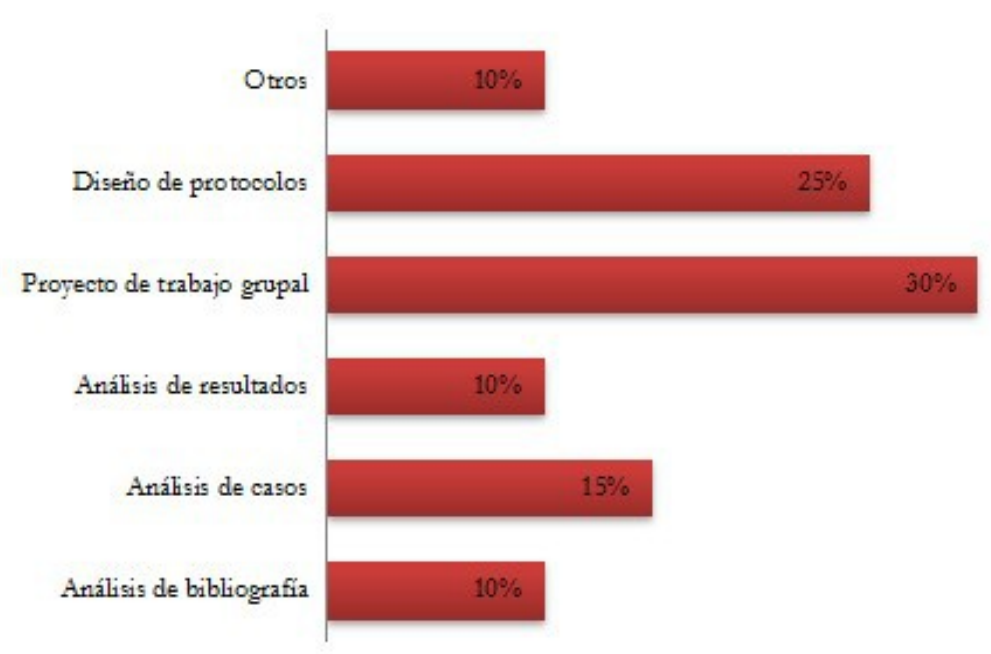

Figura 9. Estrategias de evaluación

\section{La evolución del conocimiento didáctico sobre el trabajo científico}

El conocimiento didáctico sobre la naturaleza de la ciencia y más específicamente, sobre el trabajo de investigación científica que poseía este grupo de docentes podría describirse como un conocimiento dual con una cara visible relacionada con el propio conocimiento científico obtenido a partir de una investigación sistemática aplicando el método científico centrada en la experimentación, el planteo de hipótesis y la discusión de resultados; y otra cara oculta, relativa al proceso mismo de la investigación que incluye acciones y deseos más terrenales como las dificultades, el esfuerzo, la escasez de recursos o las exigencias e injusticias del sistema. Este último aspecto no fue considerado por los docentes como algo a ser compartido con sus estudiantes, manteniendo la imagen de una ciencia construida a partir de verdades y certezas, que luego critican como ideas presentes en sus estudiantes. Podríamos decir entonces que el conocimiento didáctico al comienzo de la secuencia estuvo en un punto intermedio entre las concepciones empiristas de la ciencia y la visión de transmisión recepción del aprendizaje sostenida por profesores chilenos (Martínez y González 2014) y la excelencia de los casos estudiados por Jaruta y Medina (2012).

Del análisis de las respuestas obtenidas durante el debate en plenario surgieron además algunas nociones para profundizar la discusión. En primer lugar, observamos serias limitaciones para responder a las preguntas del cuestionario por parte de los docentes por el uso de subjetivemas de difícil categorización ("contenido abrumador") o lo acotado del vocabulario pedagógico (Jackson, 2002). Por otra parte, al igual que la literatura se observó la aparición de ciertas contradicciones en el discurso de los docentes (Caballero y Bolívar 2015); mientras por un lado, el trabajo científico y su metodología parecen ser relevantes para el futuro desempeño laboral, muchos consideraron que no era el momento oportuno para enseñarlo o temían desviarse de lo pautado. Cabría preguntarnos entonces ¿en qué momento deberían enseñarse contenidos propios de la actividad profesional de la carrera? ¿Tal vez en un curso de posgrado? ¿O es algo que los graduados deberían averiguar por su propia cuenta?

Otro ejemplo paradójico fue el problema de la falta de interés (entre otras faltas de...) de los estudiantes (universitarios, personas mayores de dieciocho años, que eligieron libremente cursar estas titulaciones y no otras). Si así fuera, en ningún caso los docentes asumieron una postura comprometida al respecto, ofreciendo ideas, sugerencias o alternativas para generar ese interés en sus alumnos. Es decir, no parece haber una reflexión sobre la propia práctica al 
considerar las limitaciones que señalan en sus alumnos. El trabajar con la secuencia didáctica propuesta permitió una revisión metacognitiva de estos conceptos con relación al desempeño de los profesores del grupo.

Si bien la naturaleza del trabajo científico quedaba implícita en el desarrollo de las actividades de enseñanza no se detectó la necesidad de hacerla explícita como tampoco los aspectos relacionados con la historia de la disciplina que enseñan. Otro punto que tampoco surgió en las respuestas iniciales de los docentes y es central para el trabajo científico, lo constituye la gestión del error y del fracaso.

Todos estos aspectos fueron expuestos al momento de interpelar las visiones sostenidas por el grupo. Como dato alentador se puede señalar el hecho de que después de la discusión en los grupos, fueron los propios docentes los que detectaron estas incongruencias o inconsistencias y debatieron sobre alternativas posibles para modificar esas situaciones. De este modo, la secuencia implementada promovió el proceso formativo al ayudar al profesorado a desarrollar concepciones teóricas sobre la docencia más centradas en el aprendizaje del alumnado reflexionando sobre diversas estrategias para mejorar la calidad de los aprendizajes (Caballero y Bolívar 2015).

\section{Conclusiones y perspectivas}

La investigación en didáctica de la ciencia cobra sentido cuando los resultados que aporta permiten la comprensión de los fenómenos que aborda y a la vez, da pistas para revisar las prácticas y corregir el rumbo cuando es necesario.

En este caso, la secuencia de actividades planteada de manera recursiva, con diferentes instancias de trabajo presencial y tiempo suficiente para la discusión y la reflexión, favoreció la revisión de las posturas y los conocimientos de los profesores a través del cuestionamiento de sus ideas iniciales y su reformulación. Los resultados mostraron que el conocimiento profesional de los docentes está condicionado por la cultura dominante, viendo a los contenidos más como fines en sí mismos que como medios para interpretar (y transformar) el mundo.

Si bien los docentes universitarios, por su rol como investigadores, conocen aspectos de la naturaleza de la ciencia y del trabajo científico, no suelen incluirlos en su enseñanza. Al confrontarlos con preguntas específicas sobre el tema, a través de la actividad implementada, pudieron explicitar sus concepciones sobre la naturaleza y epistemología de la ciencia lo que propició la discusión enriquecida por los aportes teóricos. La propuesta de trabajo en pequeños grupos y la discusión en plenario favoreció el intercambio de ideas sin herir susceptibilidades o sentimientos personales que suelen aparecer en instancias de capacitación docente ante la exposición de las propias creencias y concepciones.

Estos resultados intentan aportar al desarrollo de la didáctica universitaria de las ciencias como área particular de conocimiento, promoviendo el debate, siguiendo a Imbernón (op. cit., p. 388) cuando señala: «...la formación del profesorado universitario, inicial y permanente, es necesaria e imprescindible en una universidad que pretende mirar hacia un futuro diferente con una nueva forma de enseñar. Una nueva universidad que supere los viejos esquemas y las antiguas ideologías académicas sobre la docencia predominantes desde hace siglos, y, hoy día, mayoritariamente obsoletas.»

\section{Agradecimientos}

Este trabajo fue realizado en el marco de los Proyectos UBACYT No 20020130100073BA (2014-2017), CONICET-PIP No 11220130100609CO (2014-2016) y PICT-ANPCYT-2015-0044. 


\section{Referencias}

Acevedo J.A. (2009a) Conocimiento didáctico del contenido para la enseñanza de la naturaleza de la ciencia (I): El marco teórico. Revista Eureka sobre Enseñanza y Divulgación de las Ciencias, 6 (1), 21-46.

Acevedo J.A. (2009b) Conocimiento didáctico del contenido para la enseñanza de la naturaleza de la ciencia (II): Una perspectiva. Revista Eureka sobre Enseñanza y Divulgación de las Ciencias, 6 (2), 164-169.

Acevedo J.A. (2010). Formación del profesorado de ciencias y enseñanza de la naturaleza de la ciencia. Revista Eureka sobre Enseñanza y Divulgación de las Ciencias, 7 (3), 653-660.

Acevedo J.A., Vázquez A., Martín M., Oliva J.M., Acevedo P., Paixão M.F., Manassero M.A. (2005) Naturaleza de la Ciencia y Educación Científica para la Participación Ciudadana. Una Revisión Crítica. Revista Eureka sobre Enseñanza y Divulgación de las Ciencias, 2 (2), 121-140.

Acevedo-Díaz J., García-Carmona A. (2016) «Algo antiguo, algo nuevo, algo prestado». Tendencias sobre la naturaleza de la ciencia en la educación científica. Revista Eureka sobre Enseñanza y Divulgación de las Ciencias, 13 (1), 3-19.

Álvarez-Rojo V., Romero S., Gil-Flores J., Rodríguez-Santero J., Clares J., Asensio I., delFrago R., García-Lupión B., García-García M., González-González D., Guardia S., Ibarra M., López-Fuentes R., Rodríguez-Gómez G., Salmeron-Vilchez P. (2011) Necesidades de formación del profesorado universitario para la adaptación de su docencia al Espacio Europeo de Educación Superior (EEES). RELIEVE 17 (1), art. 1.

Barragués J.I., Garmendia M., Guisasola J., Zuza K. (2014) Proyecto de formación del profesorado universitario de Ciencias, Matemáticas y Tecnología, en las metodologías de Aprendizaje Basado en Problemas y Proyectos. Enseñanza de las Ciencias 32 (2), 113 129. doi:10.5565/rev/ensciencias.911

Berry A., Depaepe F., van Driel J. (2016) Pedagogical Content Knowledge in Teacher Education. En: J. Loughran y M.L. Hamilton (Eds.), International Handbook of Teacher Education, Volume 1 (pp. 347-386), Singapur: Springer.

Briceño J.J., Benarroch, A. (2013). Concepciones y creencias sobre ciencia, aprendizaje y enseñanza de profesores universitarios de ciencias. Revista electrónica de investigación en educación en ciencias, 8 (1), 24-41.

Briceño J.J., Benarroch, A., Marín N. (2013) Coherencia epistemológica entre ciencia, aprendizaje y enseñanza de profesores universitarios colombianos. Comparación de resultados con profesores chilenos y españoles. Enseñanz̧a de las Ciencias 31(2), 55-74. doi: $10.5565 / \mathrm{rev} / \mathrm{ec} / \mathrm{v} 31 \mathrm{n} 2.741$

Caballero K., Bolívar A. (2015) El profesorado universitario como docente: Hacia una identidad profesional que integre docencia e investigación. REDU. Revista de Docencia Universitaria, 13 (1), 57-77. doi: 10.4995/redu.2015.6446

De Miguel M. (2003). Calidad de la enseñanza universitaria y desarrollo profesional del profesorado. Revista de Educación 331, 13-34.

Demirdöğen B., Hanuscin D., Uzuntiryaki-Kondakci E., Köseoğlu F. (2016) Development and Nature of Preservice Chemistry Teachers' Pedagogical Content Knowledge for Nature of Science. Research in Science Education, 46 (4), 575-612. 
Faikhamta C. (2013) The development of in-service science teachers' understandings of and orientations to teaching the nature of science within a PCK-based NOS course. Research in Science Education, 43 (2), 847-869.

Furió C.J. (1994) Tendencias actuales en la formación del profesorado de ciencias. Enseñanz̧a de las Ciencias 12 (2), 188-199.

García-Martínez A., Izquierdo M. (2014) Contribución de la Historia de las Ciencias al desarrollo profesional de docentes universitarios. Enseñanza de las Ciencias 32 (1), 265281. doi: $10.5565 / \mathrm{rev} /$ ensciencias. 758

Glaser B.G., Strauss A.L. (1967) The discovery of grounded theory: strategies for qualitative research, Chicago: Aldine.

Grisales-Franco L. (2012) Aproximación histórica al concepto de didáctica universitaria. Educación y Educadores, 15 (2), 203-218.

Huertas J.A., Montero I. (2001) La interacción en el aula. Aprender con los demás. Buenos Aires: Aique.

Imbernón F. (2011) La formación pedagógica del docente universitario. Educação (UFSM), 36 (3), 387-396. doi: 10.5902/198464442970

Jackson P.W. (2002) Práctica de la Enseñanz̧a. Avellaneda: Amorrurtu.

Jaruta B. y Medina J.L. (2012) Saberes docentes y enseñanza universitaria. Estudios sobre Educación, 22, 179-198.

Jiménez-Tenorio N., Oliva J.M. (2016) Aproximación al estudio de las estrategias didácticas en ciencias experimentales en formación inicial del profesorado de Educación Secundaria: descripción de una experiencia. Revista Eureka sobre Enseñanza y Divulgación de las Ciencias, 13 (1), 121-136. doi: 10.25267/Rev_Eureka_ensen_divulg_cienc.2016.v13.i1.09

Latorre A. (2005) La investigación-acción. Conocer y cambiar la práctica educativa. Barcelona: Graó.

Lederman N.G. (2006) Research on nature of science: reflections on the past, anticipations of the future. Asia-Pacific Forum on Science Learning and Teaching, 7 (1), 1-11.

Lederman N.G., Antink A., Bartos S. (2012) Nature of Science, Scientific Inquiry, and SocioScientific Issues Arising from Genetics: A Pathway to Developing a Scientifically Literate Citizenry. Science \& Education, 23 (2), 285-302.

Liesa E., Castelló M., Becerril, L. (2018) Nueva escuela, ¿nuevos aprendizajes? Revista de Estudios y Experiencias en Educación, Número Especial 2, 15-29.

Lorenzo M.G. (2009) Cuando formar grupos es contenido de este curso. Educación en la Quimica, 15 (1), 54-65.

Lorenzo M.G. (2012) Los formadores de profesores: el desafío de enseñar enseñando. Profesorado: revista de curriculum y formación del profesorado, 16 (2), 295-312.

Lorenzo M.G. (2017) Enseñar y aprender ciencias y sobre las ciencias en la universidad. Nuevos escenarios para la interacción entre docentes y estudiantes. Educación y Educadores, 20 (2), 249-263.

Lorenzo M.G., Daza S. y Garritz, A. (Eds,) (2014) Conocimiento Didáctico del Contenido. Una perspectiva Iberoamericana. Saarbrücken, Alemania: Académica Española.

Lorenzo M.G., Farré A.S. (2014) Epistemología, Historia y Filosofía de las ciencias: Un puente entre la investigación didáctica y la enseñanza de las ciencias. En: M. Arellano, C. 
Merino y A. Adúriz-Bravo (Eds.), Avances en Didáctica de la Química: Modelos y lenguajes (pp. 167-182), Valparaíso, Chile: Pontificia Universidad Católica de Valparaíso.

Loughran, J., Mulhall P., Berry, A. (2004) In search of pedagogical content knowledge in science: Developing ways of articulating and documenting professional practice. Journal of Research in Science Teaching, 41(4), 370-391.

Magnusson S., Krajcik, J., Borko, B. (1999) Nature, sources, and development of pedagogical content knowledge for science teaching. En J. Gess-Newsome y N.G. Lederman (Eds.) Examining Pedagogical Content Knowledge. The Construct and its Implications for Science Education (pp. 95-132). Holanda: Kluwer Academic Publishers.

Martín del Pozo R., Porlán R., Rivero A. (2005) Secuencias formativas para facilitar el aprendizaje profesional. Revista Electrónica Interuniversitaria de Formación del Profesorado, 8 (4), 1-4.

Martínez C. y González C. (2014) Concepciones del profesorado universitario acerca de la ciencia y su aprendizaje y cómo abordan la promoción de competencias científicas en la formación de futuros profesores de Biología. Enseñanza de las Ciencias, 32 (1), 51-81. doi: $10.5565 / \mathrm{rev} /$ ensciencias. 852

Mas O. (2011) El profesor universitario: sus competencias y formación. Profesorado. Revista de Curriculum y Formación de Profesorado, 15 (3), 195-211.

Mellado V. (1999) La Formación Didáctica del Profesorado Universitario de Ciencias Experimentales. Revista Interuniversitaria de Formación del Profesorado, 34, 231-241.

Moreno T. (2011) Didáctica de la Educación Superior: nuevos desafíos en el siglo XXI. Revista Perspectiva Educacional, 50 (2), 26-54.

Perrenoud P. (2007) Desarrollar la práctica reflexiva en el oficio de enseñar. Barcelona: Graó.

Shulman L.S. (1986) Those who understand: knowledge growth in teaching. Educational Researcher, 15, 4-14.

Vázquez B., Jiménez R., Mellado V. (2007) El desarrollo profesional del profesorado como integración de la reflexión y la práctica. La hipótesis de la complejidad. Revista Eureka sobre Enseñanza y Divulgación de las Ciencias, 4 (3), 372-393.

Zabalza M.A. (2007) Competencias docentes del profesorado universitario. Madrid: Narcea. 